\title{
Use of Angular Azachlorophenothiazine as Organic Halide of Choice in Suzuki Coupling
}

\author{
A. O. IJEOMAH ${ }^{1 *}$ and U. C. OKORO ${ }^{2}$ \\ ${ }^{1}$ Department of Chemistry, University of Agriculture, Makurdi, Nigeria \\ ${ }^{2}$ Department of Chemistry, University of Nigeria, Nsukka, Nigeria \\ tinaijeomah@gmail.com
}

Received 6 May 2018 / Accepted 1 June 2018

\begin{abstract}
Chloro-10-methyl-1,11-diazaphenothiazin-5-one was synthesized and used in crosscoupling of arylboronic acids using Suzuki protocol under nickel complex catalysis to furnish intense coloured heterocycles. Structures assigned to the synthesized were supported by spectra and analytical data obtained.
\end{abstract}

Keywords: Phenothiazinone, Suzuki reaction, Cross-coupling, Nickel catalysis

\section{Introduction}

The transition metal-catalyzed cross-coupling of organoboron compounds with organic halides or pseudohalides in the presence of a base is known as Suzuki coupling or SuzukiMiyaura reaction. It is a powerful method for the formation of both aryl and alkyl carboncarbon bond ${ }^{1}$. Although there are several other cross-coupling reactions such as Stille, Sonogashira, Kumada, Negishi, Heck etc., available for the purpose, the Suzuki Miyuara reaction has proven to be more popular as a result of its many advantages which include; mild reaction condition, tolerance of wide range of functional group on the coupling partners and the environmental friendliness of boronic acid and their by- products $^{2}$. Suzuki coupling has been used in the syntheses of many useful compounds ${ }^{3}$. Diverse bases such as $\mathrm{K}_{2} \mathrm{CO}_{3}$, $\mathrm{Cs}_{2} \mathrm{CO}_{3}, \mathrm{TiOH}, \mathrm{AcONa}, \mathrm{Ba}(\mathrm{OH})_{2}$, etc. and solvents like DMA, DME/ $\mathrm{H}_{2} \mathrm{O}, \mathrm{EtOH}, \mathrm{MeOH}$, $\mathrm{H}_{2} \mathrm{O}$ etc., have employed in this reaction ${ }^{4,5}$. There are diverse numbers of organic halides available as coupling partners of which only a few have been utilized. Aza halophenothiazines used in this work have not received much attention as organic halide of choice in Suzuki coupling. The phenothiazine moieties are of great importance in medicine 6 and industry ${ }^{7}$ hence the syntheses of new derivatives has remained an area of intense interest for organic chemists. 


\section{Experimental}

Melting points of the synthesized compounds were determined by the use of Fischer Johns electro-thermal melting point apparatus in open capillaries and are uncorrected. Ultraviolet visible spectra were done on scan Buffer 16 CEUL CE 9050 spectrophotometer using matched $1 \mathrm{~cm}$ quartz cells in Department of Pure and Industrial Chemistry, University of Nigeria, Nsukka. The absorption maxima were given in nanometer $(\mathrm{nm})$. Infrared spectra were recorded with FTIR-8400s spectrophotometer in NARICT, Zaria, Nigeria, using KBr discs and the absorption values were given in per centimeter $\left(\mathrm{cm}^{-1}\right)$. Nuclear magnetic resonance was determined with Varian NMR-Mercury Spectrometer in Central Science Laboratory Obafemi Awolowo University, Ile -Ife Nigeria and chemical shift $\delta$ is reported in ppm. MS spectra were obtained from GCMS-QP2010 plus Shimadzu, in NARICT, Zaria, Nigeria. Elemental analyses were done on a CE 440 elemental Analyzer at the Central Science Laboratory University of Cairo, Egypt. Most of the chemicals were purchased from Aldrich chemical company and were used without further purification. Column chromatography was done using silica gel (mesh 60-80)

\section{Dichloro-5, 8-quinolinequinone (2)}

5-Amino-8-hydroxyquinoline $1(8.0 \mathrm{~g}, 0.05 \mathrm{~mol})$ was dissolved in $50 \mathrm{~cm}^{3}$ of concentrated $\mathrm{HCl}$ with vigorous stirring at room temperature. An aqueous solution of potassium chlorate $(7.5 \mathrm{~g}, 0.06 \mathrm{~mol})$ in $50 \mathrm{~cm}^{3}$ of water was gradually added to it. It was stirred at $50{ }^{\circ} \mathrm{C}$ for $2 \mathrm{~h}$ and then at room temperature for $3 \mathrm{~h}$. Cold water was added and the precipitate was filtered out. This crude product was re-crystallized from ethanol to obtain a bright yellow powder $(6.8 \mathrm{~g}, 60 \%)$ which melted at $217-219{ }^{\circ} \mathrm{C}$ (Lit $\left.220-222^{\circ} \mathrm{C}\right)^{8}$. UV $\lambda_{\max } 344.8, \mathrm{IR}(\mathrm{KBr}) v_{\max }$; $1640 \mathrm{~cm}^{-1}, 1579 \mathrm{~cm}^{-1}, 1505 \mathrm{~cm}^{-1}, 1458 \mathrm{~cm}^{-1}$ and $793 \mathrm{~cm}^{-1}$.

\section{2-Amino-6-methylpyridine-3-thiol (4)}

2-Amino-6-methyl-3-thiocyanatopyridine $(3.3 \mathrm{~g}, 0.02 \mathrm{~mol})$ in a $250 \mathrm{~cm}^{3}$ round bottle flask was added sodium sulphite $(2.0 \mathrm{~g}, 0.01 \mathrm{~mol})$ and potassium hydroxide solution $\left(30 \mathrm{~cm}^{3}, 20 \%\right)$ and refluxed for $8 \mathrm{~h}$. The dark brown solution was treated with activated charcoal and heated and filtered. The filtrate was cooled, neutralized with cold acetic acid and chilled overnight. The crude product was re-crystallized from methanol-acetone mixture to obtain a light yellow solid $\left(2.1 \mathrm{~g} \mathrm{75 \% )}\right.$ which melted at $244{ }^{\circ} \mathrm{C}(\text { Lit } 245)^{9}$. IR(KBr) $v_{\max }\left(\mathrm{cm}^{-1}\right), 3255,3078$ and $1431 \mathrm{~cm}^{-1}$.

\section{6-Chloro-10-methyl-1,11-diazabenzo[a]phenothiazin-5-one (5)}

2-Amino-6-methylpyridine-3-thiol $(0.7 \mathrm{~g}, 5.0 \mathrm{mmol})$ was suspended in $40 \mathrm{~cm}^{3}$ benzene and $5 \mathrm{~cm}^{3}$ of DMF in a $250 \mathrm{~cm}^{3}$ two-necked flask equipped with a reflux condenser, thermometer and magnetic stirring bar. Sodium acetate $(0.7 \mathrm{~g}, 10 \mathrm{mmol})$ was added and refluxed for 45 min. 6, 7-Dichloro-5, 8-quinolinequinone $(1.5,5.0 \mathrm{mmol})$ was added, refluxed for $6 \mathrm{~h}$ and filtered hot. This crude product was subjected to column chromatography using benzene-methanol as eluent. The first yellowish fraction was discarded. The next fraction of intense reddish compound of 6-chloro-10-methyl-1,11-diazabenzo[a]phenthiazin-5-one 9 $(0.9 \mathrm{~g}, 56.3 \%)$ decomposes at $298{ }^{\circ} \mathrm{C}, \mathrm{UV}(\mathrm{EtOH}) \lambda_{\max } 291$ and $498 \mathrm{~nm}, \mathrm{IR}(\mathrm{KBr}) v_{\max } 3078$, 1671, 1614, 1543, 1384 and $638 \mathrm{~cm}^{-1}$. MS, $\mathrm{m} / z$ (rel.int.\%); 313( $\left.\mathrm{M}^{+}, 20 \%\right), 278(-\mathrm{Cl}, 40 \%)$, 263(-Me,10\%). ${ }^{1} \mathrm{H}$ NMR (DMSO) $;$ 7.9(d,2H,arom), 7.8(m,1H,arom), 7.7(m,1H,arom), $7.5\left(\mathrm{~m}, 1 \mathrm{H}\right.$,arom) and $2.6\left(\mathrm{~s}, 3 \mathrm{H}\right.$,methyl)ppm. ${ }^{13} \mathrm{C}$ NMR (DMSO) $\delta 185,135.0,131.0,125.0$, 40.0, ppm. Calculated for $\mathrm{C}_{15} \mathrm{H}_{8} \mathrm{~N}_{3} \mathrm{OSCl} ; \mathrm{C}, 57.42 ; \mathrm{H}, 2.55 ; \mathrm{N}, 13.40 ; \mathrm{O}, 5.10 ; \mathrm{S}, 10.21 ; \mathrm{Cl}$, 11.32. Found C, 57.50; H, 2.63; N, 13.60; S, 10.19; Cl, 11.16 . 


\section{6-Chloro-10-methyl-4,11-diazabenzo[a]phenothiazin-5-one (6)}

The last fraction eluted was reddish brown compound of 6-chloro-10-methyl-4,11diazabenzo[a]phenothiazin-5-one $10(0.35 \mathrm{~g}, 21 \%) \mathrm{mp}:>300{ }^{\circ} \mathrm{C}$. UV (EtOH) $\lambda_{\max } 291,446$ $\mathrm{nm}$ and $497 \mathrm{~nm}$. IR (KBr) $v_{\max }$ 2965, 1672, 1548 and $1384 \mathrm{~cm}^{-1}, \mathrm{MS}: m / z$ (rel.int.\%), 313( $\left.\mathrm{M}^{+}, 50 \%\right), 315(\mathrm{M}+2,15 \%), 278(-\mathrm{Cl}, 100 \%), 263(-\mathrm{Me} .30 \%) .{ }^{1} \mathrm{H}$ NMR (d $\left.\mathrm{d} M S O\right) \delta$; $8(\mathrm{~d}, 1 \mathrm{H}), 7.6(\mathrm{~d}, 2 \mathrm{H}), 7.5(\mathrm{~d}, 1 \mathrm{H}), 7.1(\mathrm{~d}, 1 \mathrm{H}) .{ }^{13} \mathrm{C} \mathrm{NMR}(\mathrm{DMSO}) \delta ; 183, \mathrm{C}=\mathrm{O}, 155, \mathrm{C}=\mathrm{N}, 135$, 125, C=C, 41, C-C(methyl).

\section{General method for the synthesis of 6-aryl-10-methyl-1,11-diazabenzo[a] phenothiazin-} 5-one $(5 a-d)$

The method reported by Inada et al., ${ }^{10}$ was adopted. In a $100 \mathrm{~cm}^{3}$ two necked flask containing a magnetic stirring bar was added $\mathrm{NiCl}_{2}\left(\mathrm{PPh}_{3}\right)_{2}(0.04 \mathrm{~g}, 0.09 \mathrm{mmol}), \mathrm{PPh}_{3}(0.05 \mathrm{~g}, 0.18 \mathrm{mmol})$, arylboronic acid $(3.9 \mathrm{mmol}), \mathrm{K}_{3} \mathrm{PO}_{4} \cdot 2 \mathrm{H}_{2} \mathrm{O}(1.9 \mathrm{~g}, 7.8 \mathrm{mmol})$ and then flushed with nitrogen gas. Toluene $15 \mathrm{~cm}^{3}$ and 6-chloro-10-methyl-1, 11-diazabenzo[a]phenothiazin-5-one $(0.92 \mathrm{~g}$, $3.0 \mathrm{mmol}$ ) were added and flushed again with nitrogen gas. The reaction mixture was stirred and refluxed at $80{ }^{\circ} \mathrm{C}$ for $4 \mathrm{~h}$. The crude products was extracted with toluene, washed with water and brine, concentrated to small bulk and finally recrystallized from ethanol to obtain high yield of the cross-coupled products.

\section{0-Methyl-6-phenyl-1, 11-diazabanzo[a]phenolhinzin-5-one (5a)}

Following the general procedure given above, a mixture of 6-chloro-10-methyl-1,11diabenzo[a] phenothiazin-5-one $(0.92 \mathrm{~g}, 3.0 \mathrm{mmol}), \mathrm{NiCl}_{2}\left(\mathrm{PPh}_{3}\right)_{2}(0.04 \mathrm{~g}, 0.09 \mathrm{mmol})$, $\mathrm{PPh}_{3}(0.05 \mathrm{~g}, 0.18 \mathrm{mmol})$ and $\mathrm{K}_{3} \mathrm{PO}_{4} \cdot 2 \mathrm{H}_{2} \mathrm{O}(1.9 \mathrm{~g}, 7.8 \mathrm{mmol})$ was refluxed in toluene for 4 hours at $80{ }^{\circ} \mathrm{C}$ gave an intense red powder $(0.93 \mathrm{~g}, 85 \%$ yield $), \mathrm{mp} 120-123{ }^{\circ} \mathrm{C}$. UV-Visible $(\mathrm{EtOH}) \lambda_{\max }(\mathrm{nm})(\varepsilon) 273$ (1748) 348 (297), 404 (222). IR (KBr) $v_{\max }\left(\mathrm{cm}^{-1}\right) ; 3059(\mathrm{C}-\mathrm{H}$, aromatic), $2928\left(\mathrm{C}-\mathrm{H}\right.$, methyl), $1636(\mathrm{C}=\mathrm{O}), 1562,1486,1430(\mathrm{C}=\mathrm{C}, \mathrm{C}=\mathrm{N}) .{ }^{1} \mathrm{H}$ NMR $\delta$; $7.50\left(5 \mathrm{H}\right.$, aromatic), 8.2- $8.3\left(3 \mathrm{H}\right.$, aromatic), $8.7\left(2 \mathrm{H}\right.$, aromatic), $3.5\left(3 \mathrm{H}, \mathrm{CH}_{3}\right) \cdot{ }^{13} \mathrm{C} \mathrm{NMR} \delta$; $182(\mathrm{C}=\mathrm{O}), 167(\mathrm{C}=\mathrm{N}), 134,126(\mathrm{C}=\mathrm{C})$. MS $(\mathrm{m} / \mathrm{z}, \%$ int $) ; 355\left(\mathrm{M}^{+}, 75\right), 340(-\mathrm{Me}, 40), 263(-$ $\mathrm{Ph}, 50)$. Calculated for $\mathrm{C}_{21} \mathrm{H}_{13} \mathrm{~N}_{3} \mathrm{OS} ; \mathrm{C}, 70.79 ; \mathrm{H}, 3.66 ; \mathrm{N}, 11.83 ; \mathrm{O}, 4.51 ; \mathrm{S}, 9.01$. Found: $\mathrm{C}$, 70.94; H, 3.50; N, 11.87; S, 9.20.

\section{0-Methyl-6-(3-chlorophenyl)-1,11-diazabenzo[a]phenothiazin-5-one (5b)}

Following the general procedure reported above, a mixture of 3-chlorophenylboronic acid (0.61 g, $3.9 \mathrm{mmol})$, 6-chloro-10-methyl-1,11-diazabenzo[a]phenothiazin-5-one (0.92 g, $3.0 \mathrm{mmol}), \mathrm{NiCl}_{2}\left(\mathrm{PPh}_{3}\right)_{2}(0.04 \mathrm{~g}, 0.09 \mathrm{mmol}) \mathrm{PPh}_{3}(0.05 \mathrm{~g}, 0.18 \mathrm{mmol})$ and $\mathrm{K}_{3} \mathrm{PO}_{4} \cdot 2 \mathrm{H}_{2} \mathrm{O}$ $(1.9 \mathrm{~g}, 7.8 \mathrm{mmol})$ was refluxed in toluene at $80{ }^{\circ} \mathrm{C}$ for 4 hours to obtain dark red powder (0.96 g, 80\% yie1d) mp 142- $43^{\circ} \mathrm{C}$. UV-Visible (EtOH) $\lambda_{\max }(\mathrm{nm})(\varepsilon) ; 265$ (1910), 370 (1040), 466 (905). IR (KBr) $v_{\max }\left(\mathrm{cm}^{-1}\right) ; 3078(\mathrm{C}-\mathrm{H}$, aromatic), 1672(C=O), $1543(\mathrm{C}=\mathrm{C}$, $\mathrm{C}=\mathrm{N}), 683(\mathrm{C}-\mathrm{Cl}) .{ }^{1} \mathrm{H}$ NMR $\delta ; 7.9(\mathrm{~d}, 1 \mathrm{H}), 7.8(\mathrm{~d}, 2 \mathrm{H}), 7.6(\mathrm{~m}, 4 \mathrm{H}), 7.5(\mathrm{t}, 2 \mathrm{H}), 2.5(\mathrm{~s}, 3 \mathrm{H})$.

${ }^{13} \mathrm{C}$ NMR $\delta ; 172(\mathrm{C}=\mathrm{O}), 160(\mathrm{C}=\mathrm{N}), 126-136(\mathrm{C}=\mathrm{C})$. MS $(\mathrm{m} / \mathrm{z}, \%$ int $) ; 389\left(\mathrm{M}^{+}, 20\right), 354$ $(-\mathrm{Cl}, 10), 339$ (-Me,50), Calculated for $\mathrm{C}_{21} \mathrm{H}_{12} \mathrm{~N}_{3} \mathrm{OSCl}$; C, 64.70; H, 3.08; N, 10.78; O, 4.11; $\mathrm{S}, 8.22 ; \mathrm{Cl}=9.11$. Found: $\mathrm{C}, 64.82 ; \mathrm{H}, 3.10 ; \mathrm{N}, 10.83 ; \mathrm{S}, 8.25 ; \mathrm{Cl}, 9.20$.

\section{0-Methyl-6-(3-bromophenyl)-1,11-diazabenzo[a]phenothiazin-5-one (5c)}

From the reaction between 6-chloro-10-methyl-1,11-benzo[a]phenothiazin-5-one (0.9 g, $3.0 \mathrm{mmol})$ and 3-nitropheylboronic acid $(0.65 \mathrm{~g}, 3.9 \mathrm{mmol})$ in presence of $\mathrm{NiCl}_{2}\left(\mathrm{PPh}_{3}\right)_{2}$ (0.03 g, $0.09 \mathrm{mmol}), \mathrm{PPh}_{3}(0.06 \mathrm{~g}, 0.18 \mathrm{mmol})$ and $\mathrm{K}_{3} \mathrm{PO}_{4} \cdot 2 \mathrm{H}_{2} \mathrm{O}(0.64,7.8 \mathrm{mmol})$ was obtained a reddish brown powder $(0.91 \mathrm{~g}, 70 \%), \mathrm{mp} 150-152{ }^{\circ} \mathrm{C}, \mathrm{UV}-\mathrm{Visible}(\mathrm{EtOH}) \lambda_{\max }(\mathrm{nm})(\varepsilon)$; 
286 (160), 353 (257), 473 (174). IR (KBr) $v_{\max }\left(\mathrm{cm}^{-1}\right) ; 1684(\mathrm{C}=\mathrm{O}), 1576,1609,1563(\mathrm{C}=\mathrm{N}$, $\mathrm{C}=\mathrm{C}) . \mathrm{MS}(\mathrm{m} / z, \%$ int $) ; 433\left(\mathrm{M}^{+}, 75\right), 435\left(\mathrm{M}^{+}+2,70\right), 418(-\mathrm{Me}, 40) .{ }^{1} \mathrm{H}$ NMR $\delta ; 7.5(2 \mathrm{H}$, aromatic), $7.65(2 \mathrm{H}$, aromatic), $7.80(2 \mathrm{H}$, aromatic), $7.85(2 \mathrm{H}$ aromatic), $4.0(3 \mathrm{H}$, methyl). ${ }^{13} \mathrm{C}$ NMR $\delta ; 181(\mathrm{C}=\mathrm{O}), 167(\mathrm{C}=\mathrm{N}$, aromatic), 113-148 $(\mathrm{C}=\mathrm{C})$. Calculated for $\mathrm{C}_{21} \mathrm{H}_{12} \mathrm{~N}_{3} \mathrm{OSBr}$; C, 58.20 H, 2.77; N, 9.70; O, 3.70; S , 7.39; Br , 18.24. Found: C, 58.15; H, $2.80 ; \mathrm{N}, 9.65 ; \mathrm{S}, 7.30 ; \mathrm{Br}, 18.50$.

\section{0-Methyl-6-(3-nitrophenyl)-1, 11-diazabenzo[a]phenothiazin-5-one (5d)}

Following the general procedure reported above, a mixture of 3-nitrophenylboronic acid (0.78 g, $3.9 \mathrm{mmol})$, 6-chloro-10-methyl-1-14-diazaphenothiazin-5-one (0.92 g, $3.0 \mathrm{mmol})$ $\mathrm{NiCl}_{2}\left(\mathrm{PPh}_{3}\right)_{2}(0.04 \mathrm{~g}, 0.09 \mathrm{mmol}), \mathrm{PPh}_{3}(0.05 \mathrm{~g}, 0.18 \mathrm{mmol})$ and $\mathrm{K}_{3} \mathrm{PO}_{4} .2 \mathrm{H}_{2} \mathrm{O}(1.9 \mathrm{~g}, 7.8$ mmol) refluxed in toluene for 4 hours at $80{ }^{\circ} \mathrm{C}$ gave the titled compound, brown powder (0.93 g, 78\% yield), mp 128-130 ${ }^{\circ} \mathrm{C}$. UV-Visible (EtOH) $\lambda_{\max }(\mathrm{nm})(\varepsilon) ; 291$ (660), 498 (166), 658 (37). IR (KBr) $v_{\max }\left(\mathrm{cm}^{-1}\right) ; 2965(\mathrm{C}-\mathrm{H}$, methyl), $1675(\mathrm{C}=\mathrm{O}), 1548,1384(\mathrm{C}=\mathrm{C}$, $\mathrm{C}=\mathrm{N})$. MS $(\mathrm{m} / \mathrm{z}, \%$ int $) ; 400\left(\mathrm{M}^{+}, 30\right), 354\left(-\mathrm{NO}_{2}, 35\right), 278(-\mathrm{Ph}, 100) .{ }^{1} \mathrm{H}$ NMR $\delta ; 7.5(2 \mathrm{H}$, aromatic), $7.6(2 \mathrm{H}$, aromatic), $7.8(2 \mathrm{H}$, aromatic), 7.9 (2H aromatic) $3.5(3 \mathrm{H}$, methyl) $113-$ $135(\mathrm{C}=\mathrm{C})$. Anal calculated for $\mathrm{C}_{21} \mathrm{H}_{12} \mathrm{~N}_{4} \mathrm{O}_{3} \mathrm{~S} ; \mathrm{C}, 63.00 ; \mathrm{H}, 3.00 ; \mathrm{N}, 14.00 ; \mathrm{O}, 12.00 ; \mathrm{S}, 8.00$. Found: C, 63.20; H, 2.94; N, 14.09; S, 7.89.

\section{Results and Discussion}

2-Amino-6-methyl-3-thiocyantopyridine (3) was converted to 2-amino-6-methyl-pyridine-3thiol (4) by alkaline hydrolysis. The IR spectrum of compound 4 showed band at $2611 \mathrm{~cm}^{-1}$ is due to S-H vib stretch. The absence of absorption band in the triple bond region of 2100-2300 $\mathrm{cm}^{-1}$ showed that the thiocyanato group has been converted to the thiol group (Scheme 1).<smiles>Cc1ccc([As])cn1</smiles>

\section{Scheme 1}

6,7-Dichloroquinolinequinone $\mathbf{2}$ which is the second key intermediate was obtained by chloroxidation of 5-amino-8-hydroxyquinoline 1 using conc $\mathrm{HCl}$ and potassium chlorate while maintaining the temperature at $55-60{ }^{\circ} \mathrm{C}$. The crude product obtained was recrystallized twice from ethanol to furnish a bright yellow compound. The IR spectrum of the compound showed band at $1639 \mathrm{~cm}^{-1}$ which has been attributed to $\mathrm{C}=\mathrm{O}$ stretching vibration. The absence of absorption band at $3200 \mathrm{~cm}^{-1}$ showed that the hydroxyl and amino groups have been oxidized (Scheme 2).<smiles>Nc1ccc(O)c2ncccc12</smiles><smiles>[Mg][Mg]C(Cl)(Cl)Cl</smiles><smiles>O=C1C(Cl)=C(Cl)C(=O)c2ncccc21</smiles>

\section{Scheme 2}

Equimolar mixture of 2-amino-6-methylpyridine-3-thiol $\mathbf{3}$ and 6,7-dichloro-5,8quinolinequinone 4 in benzene mixed with DMF was reacted in the presence of anhydrous sodium acetate to furnish the isomeric mixture which was later separated by column chromatography to obtain the regio isomers. This is represented by Scheme 3 below. 


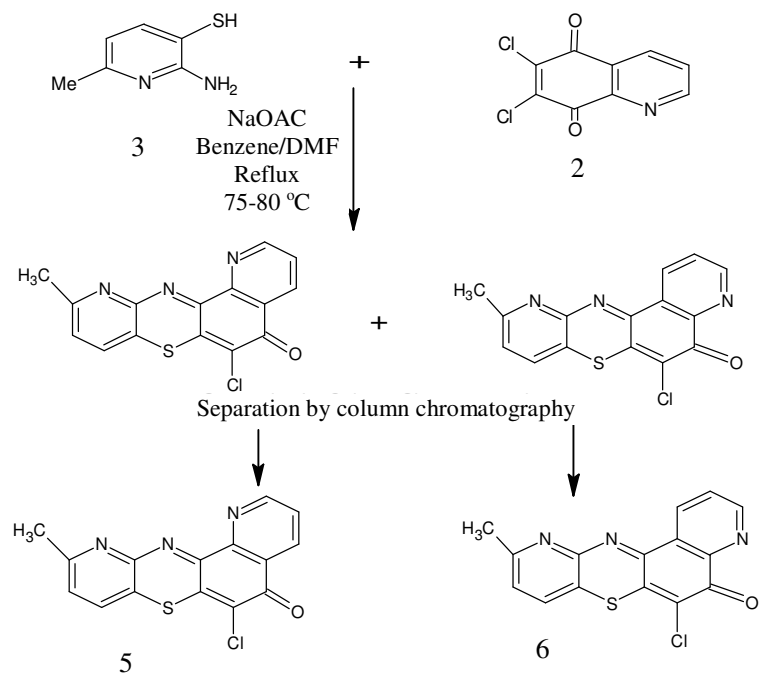

Scheme 3

Compound 5 was subjected to Suzuki Miyuara coupling with arylboronic acids to produce its 6-aryl-derivatives under nickel complex catalysis while employing $\mathrm{K}_{3} \mathrm{PO}_{4}$ as a base (Scheme 4).

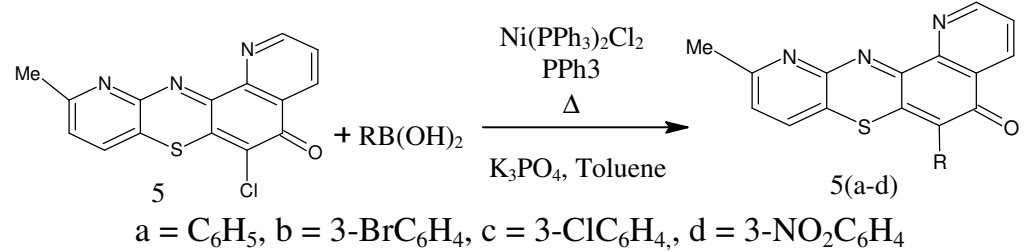

Scheme 4

The probable mechanism for this nickel-catalyzed cross-coupling is depicted in the scheme 5 below.

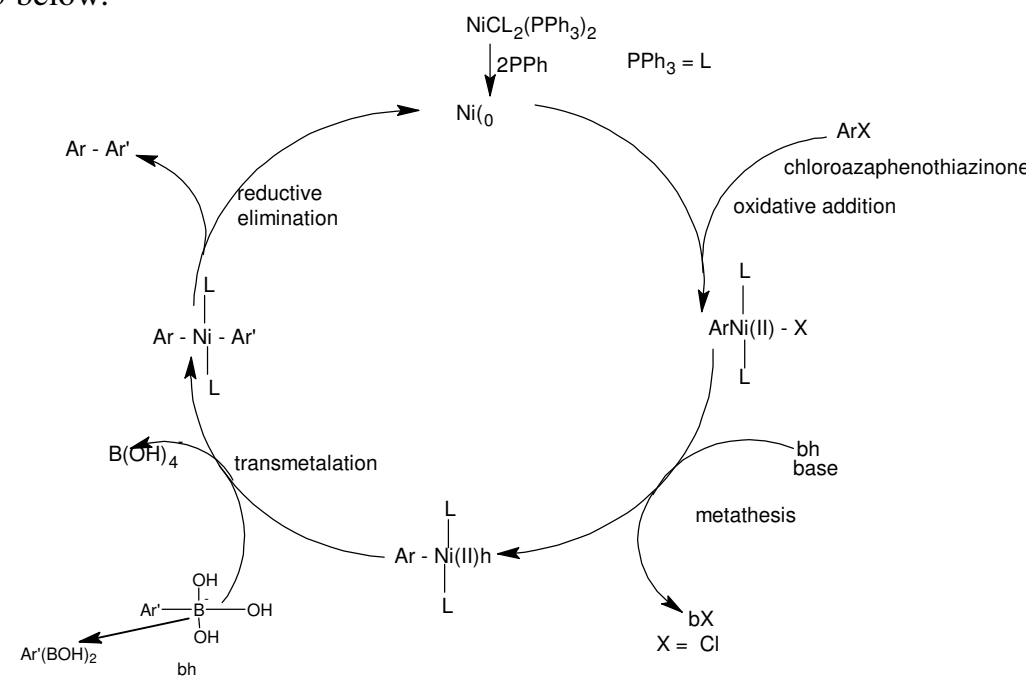

Scheme 5 
The first step is probably the reduction of $\mathrm{Ni}(\mathrm{II})$ to $\mathrm{Ni}(0)$ by the excess triphenylphosine introduced.

$$
\mathrm{NiCl}_{2}\left(\mathrm{PPh}_{3}\right)_{2}+2 \mathrm{PPh}_{3} \longrightarrow \mathrm{Ni}(0)\left(\mathrm{PPh}_{3}\right)_{4}
$$

This is followed by the oxidative addition of chlorophenothiazine to form the aryl nickel complex Ar-NiL $-\mathrm{X}$. The base displaces the chloride ion (metathesis) and also acted on the phenylboronic acid to form the boronate anion which enhances the transmetalliation. Finally through reductive elimination the product is formed and the nickel catalyst regenerated.

\section{Conclusion}

6-Chloro-10-methyl-1,11-diazaphenothiazin-5-one was synthesized and cross-coupled with arylboronic acids to furnish 6-substituted azaphenothiazinones with intense colours which can be used as vat dyes in industry.

\section{References}

1 Suzuki A, J Organomet Chem., 1999, 576(1-2), 147-168; DOI:10.1016/S0022328X(98)01055-9

2 Fu-She Han, Chem Soc Rev., 2013, 42(12), 5270-5298; DOI:10.1039/C3CS35521G

3 Yang C T, Zhen Qi Zhang, Yu Chen Liu and Lei Liu, Angew Chem Int Ed., 2011, 50(17), 3904-3907

4 Weies A N, Baker L E and Gary N K, Nature Chem., 2015, 17, 4364; DOI:10.1021/acs.orglett.5b02209

5 Lipshutz B H, Petersen T B and Abele A R, Org Lett., 2008, 10(7), 1333-1336; DOI:10.1021/ol702714y

6 Pluta k,Jelen M, Morak-Mlodawska B, Zimecki M, Artym J and Kocieba M, Pharmacol Rep., 2010, 62(2), 319-332; DOI:10.1016/S1734-1140(10)70272-3

7 Okoro U C and Ijeomah A O, International J Chem., 2006, 16(4), 245-250;

8 Johnson F, Grollman A P and Shaikh I A, J Med Chem., 1986, 29(8), 1320-1340.

9 Okafor C O, J Org Chem., 1973, 38(26), 4383-43861; DOI:10.1021/jo00965a007

10. Miyuara N and Inada K, Tetrahedron, 2000, 56(44), 8657-8660; DOI:10.1016/S0040-4020(00)00814-0 